anales de psicología, 2012, vol. $28, \mathrm{n}^{\circ} 3$ (octubre), 866-874 http://dx.doi.org/10.6018/analesps.28.3.130651
C Copyright 2012: Servicio de Publicaciones de la Universidad de Murcia. Murcia (España) ISSN edición impresa: 0212-9728. ISSN edición web (http://revistas.um.es/analesps): 1695-2294

\title{
Evaluación de los servicios de atención a familias de niños con deficiencia. Perspectiva de familiares y profesionales brasileños
}

\author{
Simone Cerqueira da Silva ${ }^{1}$, Maria Auxiliadora Dessen ${ }^{1}$ y Julio Pérez-López ${ }^{2 *}$ \\ ${ }^{1}$ Universidade de Brasília. Instituto de Psicología. Laboratorio de Desarrollo Familiar (Brasil) \\ ${ }^{2}$ Universidad de Murcia. Grupo de Investigación en Atención Temprana (España)
}

\begin{abstract}
Resumen:
Introducción.- Se investigaron los servicios ofertados y la atención dirigida a las familias desde la perspectiva de las familias de niños con deficiencia y de los profesionales de las instituciones que las atendían.

Métodos y procedimiento.- 80 participantes distribuidos en: familiares $(n=54)$ y profesionales $(n=26)$, pertenecientes a cinco instituciones que atendían a niños con discapacidad. Para la recogida de datos se utilizó un cuestionario y un modelo de entrevista semiestructurada.

Resultados.- El análisis cualitativo de las entrevistas mostró que la atención ofrecida a las familias era esporádica e informal, realizada de forma individual o en grupo. Las evaluaciones de los profesionales y de las familias aportaron puntos positivos como el apoyo emocional y las orientaciones sobre las relaciones parentales, la deficiencia y el desarrollo de los niños. En cuanto a los aspectos negativos, los expertos apuntaron que los servicios y atención a la familia no eran prioritarios, y las familias evaluaron que los servicios eran insuficientes y no atendían sus necesidades.

Discusión y Conclusiones.- Estos datos indican que las intervenciones familiares tienen como centro al niño y están basadas en el modelo del experto. Considerando la importancia de promover la atención temprana, es necesario poner en marcha cambios que sirvan para mejorar la calidad de las intervenciones familiares.
\end{abstract}

Palabras clave: Intervención familiar; apoyo familiar; atención temprana; discapacidad; infancia.

\section{Introducción}

La teoría ha demostrado que el apoyo familiar y las competencias de cada miembro de la familia son recursos importantes que influyen en el modo en que un niño convive con su discapacidad (Pereira-Silva y Dessen, 2001) y que el desajuste del niño está más relacionado con la forma en la que la familia convive con él que con sus propios comportamientos (Robbins, Szapocznik, Tejeda, Samuels, Ironson y Antoni, 2003). Los niños con discapacidad, por ejemplo, pueden llegar a presentar problemas no en función de la discapacidad en sí misma, sino de la inadecuación del comportamiento de sus progenitores, que pueden desarrollar una práctica educativa basada en la permisividad y la superprotección, algo común en estos casos.

En el ambiente familiar es donde un niño puede recibir tanto protección como convivir con riesgos para su desarrollo, los cuales se refieren con frecuencia al bajo nivel socioeconómico y a la fragilidad en los vínculos afectivos (Santos y Graminha, 2005), dando lugar a alteraciones en la solución de problemas, lenguaje, memoria y habilidades sociales. Por otro lado, los niños con algún tipo de necesidades educativas

* Dirección para correspondencia [Correspondence address]: Julio Pérez-López. Departamento de Psicología Evolutiva y de La Educación. Facultad de Psicología. Universidad de Murcia. Campus de Espinardo. 30100 Murcia (España). E-mail: juliopl@um.es
Title: Evaluation of the care services for families of disabled children. Perspective of Brazilian family members and professionals

Abstract:

Introduction.- This study aims to investigate the services offered and the attention given to families from the perspective of the families of disabled children and professionals of the Brazilian institutions that attended them. Methods and procedures.- There were 80 participants, divided into two categories: family members $(n=54)$ and professionals $(n=26)$, belonging to five institutions catering to children with disabilities. For data collection, a questionnaire and a semi-structured interview model were used.

Results.- The qualitative analysis of the interviews shows that the care offered to families was mostly sporadic and informal, conducted by an individual or group. Evaluations of professionals and families indicated that there were some positive points such as emotional support and guidance on parental relationships and children's deficiency and development. As for the negative side, the experts pointed out that the services and care for the family were not a priority, and the families assessed those services as inadequate and not enough to fulfil their needs.

Discussion and Conclusions.- The results indicate that family interventions are focused on the child in isolation and based on a model centred on the expert. Considering the importance of promoting early intervention, it is necessary to implement changes that improve the quality of family interventions.

Key words: Family intervention; family support; early intervention; disability; infancy.

especiales también pueden ser considerados como factor de riesgo para el desarrollo de la familia en la medida en que exigen más cuidados y atención, alterando, muchas veces, las relaciones familiares.

De acuerdo con Brazelton y Greenspan (2002), la familia se puede convertir en un ambiente facilitador y saludable cuando atiende las necesidades esenciales de sus hijos. Para ello, los padres necesitan estar atentos a las necesidades tanto individuales como de protección física y seguridad del niño, garantizando un cuidado continuo. Esto se consigue mediante cuidadores sensibles y constantes a lo largo de su ciclo vital. Por otro lado, los patrones familiares que perjudican el cuidado sostenible, acogedor y sensible pueden llevar a un problema significativo en las capacidades cognitivas y emocionales del niño y, en muchas situaciones, agravarán las condiciones de su discapacidad.

Teniendo en cuenta la importancia y contribución de la familia para la promoción del desarrollo de los menores, en especial aquellos que se encuentran en situación de riesgo o que presentan algún tipo de discapacidad, es fundamental que las estrategias utilizadas por los profesionales incluyan a la familia y no, simplemente, a los niños (Pereira-Silva y Dessen, 2005; Pérez-López, 2008). Para alcanzar niveles satisfactorios de adaptación a la situación de discapacidad, las familias demandan mayor apoyo, cuidado, información, acogida y orientación en las tareas de desarrollo familiar, presen- 
tando, por consiguiente, necesidades diferentes y especiales (Robles y Romero, 2011; Silva y Elsen, 2006).

Además de tener que cumplir, como cualquier persona, las tareas normativas de la vida familiar, estas tareas requieren estrategias especiales para poder afrontar las características de los niños y su influencia en los subsistemas familiares: parental, conyugal y fraternal. Esto significa que para garantizar un ambiente potenciador, sensible a las necesidades individuales, seguro, organizado y protector para los niños, esas familias necesitan, incluso antes del nacimiento de sus bebés, información, orientación y una amplia red de apoyo social (Brazelton y Greenspan, 2002; Oliveira, 2007).

En este sentido, diversos estudios han mostrado la importancia de las diferentes contribuciones de la red de apoyo social, como un factor protector importante para las familias que tienen niños con discapacidad (Gomes y Bosa, 2004; Nuñez, 2003; Paniagua, 2004). El apoyo social es definido como un conjunto de apoyos, tanto informales (vecinos, amigos, iglesia) como formales (profesionales), que tienen un impacto directo o indirecto sobre las familias, los padres y los niños (Dunst, 2000). Duvdevany y Abboud (2003) sugirieron que las madres participen en programas especiales que las capaciten para estar más implicadas y tener más conciencia de sus derechos, así como para enfrentarse mejor a las dificultades adversas y a las necesidades especiales de sus hijos.

Una de las alternativas relevantes de apoyo son los programas de intervención familiar. A pesar del creciente interés por promover la participación de la familia en estos programas, cuya importancia no es nueva (Aiello, 2004; Campos y Garcia, 2007), los programas orientados a las familias ven lentamente ampliada su relevancia. Fue en los inicios de la década de los 90 cuando la figura de los profesionales, y los modelos de intervención centrados en los profesionales, comenzaron a ceder espacio a la participación más efectiva de las familias, pasando a fortalecerse los modelos de intervención centrados en las familias (Dunst, Boyd, Trivette y Hamby, 2002).

En los modelos centrados en los profesionales, característicos de la década de los 70 y 80, eran los profesionales los que definían todo el proceso de orientación y las familias eran vistas como menos capaces que los profesionales para elegir y tomar decisiones en cuanto a sí mismas y a sus hijos (Dunst et al., 2002); por tanto, el papel de las familias era aceptar y seguir lo impuesto por los profesionales. Por el contrario, las propuestas de trabajo con familias, basadas en el modelo centrado en la familia, se fundamentan principalmente, en el énfasis sobre la fortaleza/recursos de la familia, y no en sus dificultades, destacando la necesidad de dejar que la familia escoja y tenga control sobre los recursos deseados, generando, así mismo, la colaboración entre ella y los profesionales (Bruder, 2000).

Con esta nueva perspectiva (modelo centrado en la familia), las familias dejan de ser vistas como pasivas y dependientes de los profesionales para ser consideradas activas. Sus conocimientos y habilidades son realzados en una relación de colaboración y no tanto de dependencia (Dunst et al., 2002). Sobre ese enfoque de trabajo, se espera que la familia vaya mejorando sus capacidades y habilidades para dar respuesta a las necesidades de sus hijos que, a su vez, reciben apoyo profesional y de sus propias familias, beneficiándose de la interacción, vinculación afectiva y dedicación de su familia (de Linares y Rodríguez, 2004).

A pesar del reconocimiento de la importancia de la familia en los programas de intervención, en la historia de la evolución de los programas, permanecen algunas dudas y desafíos sobre la implementación y la política de modelos centrados en la familia. En la literatura se han citado numerosos problemas, principalmente relacionados con la aplicación de los resultados de investigación a la práctica de la intervención (ver, por ejemplo, Pereira-Silva y Dessen, 2005). Profesionales y equipos técnicos no siempre han usado los resultados de las investigaciones en las prácticas centradas en las familias, así como, tampoco han valorado esa filosofía de trabajo (Bruder, 2000, 2010).

En Brasil, los profesionales todavía tienen, hasta hoy, muchas dificultades para la implementación de estos programas (Oliveira y Braga, 2004). Por ejemplo, el estudio de Santos (1993), realizado hace 18 años, parece mostrar una realidad que todavía está presente. La autora identificó que en catorce instituciones de rehabilitación para niños con discapacidad, solamente siete proporcionaban atención a las familias de los niños, y sólo se hacía con las madres. Constató que todavía, los profesionales, se enfrentan a diversas dificultades para realizar este tipo de intervención, principalmente relacionadas con su falta de preparación profesional y con la falta de información, por parte de la dirección de esas instituciones, en cuanto a esa modalidad de atención.

A pesar de ello, las dificultades tanto para implementar los trabajos con las familias como para alcanzar los resultados propuestos suceden, en parte, debido a la tendencia a no considerar, en el planteamiento de tales intervenciones, el funcionamiento específico de las familias de acuerdo con el tipo de discapacidad del niño. Kreutz y Bosa (2009) afirman que familias de niños con deficiencia visual tienen demandas que deben ser tenidas en cuenta cuando se planifican esos programas. Una de ellas se refiere al hecho de que los profesionales siempre se comportan con ellos como si fuesen profesores o terapeutas del niño, con el objetivo constante de enseñarles cómo tratar al niño y descuidando sus necesidades emocionales. Para estas autoras, son escasas las informaciones proporcionadas tanto sobre el desarrollo del niño con deficiencia visual como sobre la eficacia de los programas de intervención con ellos.

Por tanto, y a pesar del reconocimiento de la importancia del trabajo con la familia del niño con discapacidad o en situación de riesgo, se hace necesario que los estudios empíricos identifiquen y describan cuáles son los trabajos desarrollados con las familias y su eficacia para el desarrollo de los niños y del conjunto familiar. Desde este punto de vista, el presente estudio investigó los servicios ofrecidos y las atenciones dirigidas a las familias, desde la perspectiva de niños con discapacidad y sus familias, así como desde el punto de 
vista de los profesionales de las instituciones brasileñas que las atendían. No se trata de un estudio explicativo de la situación en Brasil como país, sino que trata de identificar las fortalezas y debilidades en los servicios evaluados.

\section{Método}

Este estudio implicó a 80 participantes distribuidos en dos categorías: miembros familiares $(n=54)$ y profesionales $(n=26)$, pertenecientes a cinco instituciones que atendían a niños con discapacidad. Cuatro de estas instituciones se encontraban en la ciudad de Brasilia/Distrito Federal y una en la ciudad de Bauru (estado de São Paulo). Las instituciones de Brasilia, proporcionaban atención específica a niños con deficiencia auditiva, intelectual, física o visual; y la institución seleccionada de Bauru atendía niños con todo tipo de discapacidad. La recogida de datos fue realizada en 2008 y 2009, utilizándose un cuestionario y un modelo de entrevista semiestructurada, conforme se describe a continuación.

\section{Participantes}

Los 54 miembros familiares formaban parte de 16 familias que participaron en la investigación: 16 madres, 12 padres, 10 hermanos y 16 niños con algún tipo de discapacidad. De los 26 profesionales, 16 eran especialistas de equipos técnicos, 5 coordinadores de equipos técnicos y 5 directores de instituciones de atención a niños con discapacidad.

De los 16 niños, cuyas familias aceptaron participar en el estudio, 7 tenían entre 4 y 6 años (Grupo A) y diferentes discapacidades: auditiva $(n=3)$, múltiple $(n=2)$, intelectual $(n=1)$ y física $(n=1)$; y 9 tenían más de 6 años (Grupo B), con diferentes discapacidades: auditiva $(n=1)$, intelectual $(n=3)$, física $(n=3)$ y visual $(n=2)$.

En cuanto a las madres de los niños, tenían una media de 37 años y la mayoría $(n=9 ; 56,25 \%)$ había concluido la enseñanza media. En relación a la profesión, 8 de ellas eran amas de casa, sin ejercer ninguna actividad profesional, y las demás $(n=8)$ ejercían algún tipo de trabajo profesional. Los padres de los niños tenían una media de 43 años y la mayoría $(n=7 ; 58,33 \%)$ habían concluido la enseñanza media. Los 10 hermanos que participaron en este estudio, tenían entre $7 \mathrm{y}$ 22 años. Las familias, en su mayoría $(n=14)$, pertenecían a la clase media (C) que, en Brasil, son definidas como el estrato más común y más numeroso ( $52 \%$ de la población) que, por consiguiente, no sufre dificultades económicas ni vive con un gran margen financiero (Fundação Getúlio Vargas FGV, 2010).

En cuanto a los perfiles de los profesionales, los directores de instituciones tenían una edad media de 49 años, la mayoría $(n=3)$ tenían cursos de especialización en el área que actuaban, y los de mayor edad (con 60 y 63 años) eran los que estaban trabajando desde hacía más tiempo en este cargo, exactamente, 29 y 28 años respectivamente. Los especialistas de los equipos técnicos tenían 40 años de media de edad, todos habían cursado algún tipo de Post-graduación, habiendo realizado la mayoría $(n=13)$ la especialización en su área de trabajo. Por último, los coordinadores de equipos técnicos tenían una media de edad de 45 años y ejercían el cargo en la institución con una antigüedad que variaba entre 6 meses y 5 años (sólo un coordinador trabajaba desde hacía más de 28 años).

\section{Procedimiento e instrumentos}

Este estudio fue desarrollado en dos fases. En la primera se recogieron: (a) las características de las familias, aplicando un Cuestionario de Caracterización del Sistema Familiar (Dessen, 2009), con las 16 madres. El cuestionario tenía por finalidad investigar los datos demográficos de la familia (condición socioeconómica, ocupación de la familia y nivel educativo) y los aspectos relativos a su modo de funcionamiento (distribución de actividades domésticas y de rutina familiar); y (b) características y evaluación de los servicios, así como tipos de atención familiar ofrecidos por las instituciones. Para ello, se realizaron entrevistas con cada uno de los familiares. En la segunda fase, se obtuvo información de las características de los servicios ofrecidos por las instituciones y fueron evaluados estos servicios así como la atención ofrecida a las familias. Todo ello, se realizó a través de entrevistas con los profesionales. De este modo, los niños, los familiares y los profesionales no sólo identificaron y describieron los servicios y atenciones recibidas por las familias con niños con discapacidad, sino que también expresaron su opinión sobre la calidad y los resultados de los mismos.

Los relatos de los participantes fueron analizados en base a un sistema de categorías desarrolladas de acuerdo con los procedimientos propuestos para generar sistemas integrados de categorías (Dessen y Cerqueira-Silva, 2009). Estos sistemas integrados son el resultado de un procedimiento que consiste en sucesivas clasificaciones de las verbalizaciones en categorías, temas y subtemas, siendo la fundamentación teórica del fenómeno investigado, el punto de apoyo de la estructura del sistema. Es importante resaltar que esta investigación fue aprobada por el Comité de Ética de la Facultad de Salud de la Universidad de Brasilia-UnB (no 142/2008).

\section{Resultados}

Los resultados, referentes al punto de vista de las familias y de los profesionales se exponen en dos apartados: (a) descripción de los servicios y atención familiar; y (b) evaluación de los servicios y atención familiar.

Servicios y Atención Familiar de las Instituciones: la visión de las familias y de los profesionales.

¿Cuáles fueron las impresiones de los familiares en cuanto a los servicios y atención familiar? En primer lugar, los familiares respondieron respecto a la intervención familiar ofrecida por la institución que atendía al niño en cuanto a: atenciones prestadas, actividades sociales/ocio y actividades educati- 
vas/profesionales. La Figura 1 representa las principales intervenciones familiares en las que las madres, padres y hermanos mencionaron haber participado, así como sus respectivas frecuencias.

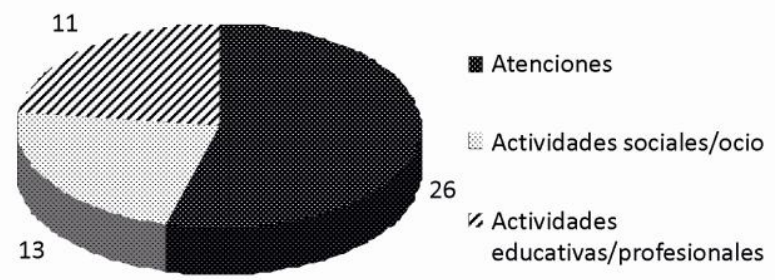

Figura 1. Principales intervenciones ofrecidas por las instituciones, según la opinión de los familiares.

Entre las atenciones realizadas $(n=26)$, la mayoría de los participantes, madres y padres, comentaron haber participado en el Grupo de Padres ( $n=9 ; 34.62 \%$ ), aunque, todos ellos se refirieron a una sola de las instituciones que ofrece este tipo de atención, tanto para los niños del Grupo A $(n=5)$ como para los niños del Grupo B $(n=4)$. Las atenciones informales $(n=7 ; 26.92 \%)$ y la Psicoterapia $(n=7$; $26,92 \%)$ también fueron comentados. Es interesante destacar que las atenciones informales fueron citadas, sobre todo, por los familiares del Grupo B $(n=6 ; 66.66 \%)$, conforme muestra el siguiente relato: Ella tiene su atención, media hora y después vuelve y cuando vuelve yo pregunto, o algunas veces ni pregunto, ellas mismas hablan... "A A.C. hizo tal cosa así, asi y asi". "Hoy en la clase no rindió porque ella estaba terca" (F3-m).

A pesar de las atenciones, las instituciones también ofrecen servicios diversos, como actividades sociales/ocio $(n=13)$ y actividades educativas / profesionales $(n=11)$. En cuanto a las actividades sociales merecen destacarse las confraternizaciones/fiestas $(n=6 ; 46.15 \%)$, sobre todo para los familiares del Grupo B $(n=4)$, y las Actividades Físicas $(n=5$; $38.46 \%$, que fueron comentadas tanto por el Grupo A $(n=3)$ como por el Grupo B $(\mathrm{n}=2)$. Las actividades educativas/profesionales $(n=11)$, también fueron comentadas por los familiares del grupo A $(n=5)$ y del grupo $B(n=6)$.

En conclusión, los datos indican que la atención ofrecida a los familiares se centra en atenderles informalmente, aunque no ocurre así con los familiares del Grupo A que cuentan con atenciones más sistematizadas y organizadas. Además de eso, fue poca la diferencia de atenciones identificadas por los familiares de los Grupos A y B, salvo que los familiares del Grupo B son quienes más participan de las Confraternizaciones/fiestas.

¿Cuáles fueron las impresiones de los profesionales en relación a los servicios de atención familiar? Los servicios y la atención familiar ofrecidos, en opinión de los profesionales, tiene como objetivos: (a) orientar sobre cuestiones de la discapacidad y del comportamiento del niño $(n=7)$; (b) orientar sobre el papel de los padres como co-terapeutas $(n=5)$; (c) reducir el sufrimiento de los padres dando apoyo emocional $(n=5)$; (d) dar un trabajo a los padres y favorecer sus ingresos económicos $(n=2)$; y (e) favorecer el acceso de los progenitores a los programas institucionales del gobierno $(n=1)$. De esta manera, el centro de atención a las familias parece ser el niño.

Para alcanzar esos objetivos, los profesionales realizan diversos tipos de servicios y de atención familiar, entre ellos, las atenciones o apoyos fueron las más comentadas $(n=22)$, también las actividades educativas y profesionales $(n=19)$ y las actividades sociales/ocio $(n=8)$. La figura 2 representa los servicios y la atención familiar ofrecidos por las instituciones, desde el punto de vista de los profesionales.

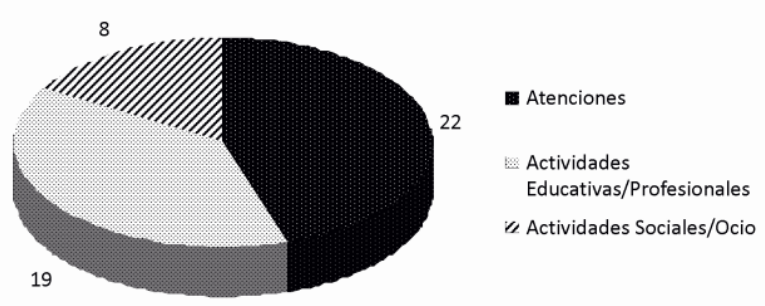

Figura 2. Servicios y atención familiar ofrecidos por las instituciones, en la opinión de los profesionales.

En lo que respecta a las atenciones $(n=22)$, las informales fueron las más citadas $(n=8 ; 36.36 \%)$, aunque los profesionales también se refirieron al Grupo de Padres $(n=5 ; 22.73 \%)$ y a la Psicoterapia $(n=5 ; 22.73 \%)$. En relación a las actividades educativas y profesionales, los Cursos de Bordado y Pintura fueron los más comentados $(n=6 ; 31.58 \%)$. En cuanto a las actividades sociales/ocio, las Confraternizaciones/fiestas $(n=3 ; 37.5 \%)$ y las Actividades Físicas $(n=2 ; 25 \%)$ fueron las más citadas.

Se aprecia que las atenciones o apoyos informales constituyen la mayoría de las ofrecidas, y que hay un énfasis en la oferta de las actividades educativas/profesionales. En este sentido, se observa la diversidad de acciones implementadas por las cinco instituciones para atender a los familiares, se percibe una gran riqueza de opciones, oscilando desde aquellas enfocadas en las necesidades emocionales y cognitivas (por ejemplo: psicoterapia, grupo de padres), hasta aquellas más enfocadas a cuestiones de orden social, profesional y ocupacional de las familias (por ejemplo: actividades físicas, cursos de bordado y pintura).

Tanto los profesionales como las familias citaron especialmente las atenciones dispensadas a los familiares (informales y grupos de padres). Entre estas dos opciones, los familiares citaron más los grupos de padres y los profesionales las informales/asistemáticas, que no estaban planificadas. Debemos observar que el modo de trabajo improvisado parece caracterizar el tipo de que los profesionales dedican a los familiares. En lo que se refiere a los servicios prestados, las familias comentaron más las actividades sociales/ocio y los profesionales las actividades educativas/profesionales. 
Evaluación de los servicios y de la atención familiar: la visión de los familiares y de los profesionales.

Los familiares y los profesionales identificaron aspectos positivos y negativos en cuanto a: (a) los propios servicios y atenciones; (b) los profesionales; (c) los familiares; y (d) a la estructura física de las instituciones

Aspectos positivos (puntos fuertes o fortalezas) de los servicios y de la atención familiar. En cuanto a los Servicios y a la Atención Familiar, fueron evaluados positivamente porque promueven conocimientos, orientaciones e informaciones, así como intercambios de experiencias, apoyo emocional (afectivo) y recompensas (materiales/objetivos). La figura 3 ilustra los aspectos positivos de los servicios y de la atención familiar, según la visión de las familias y de los profesionales.

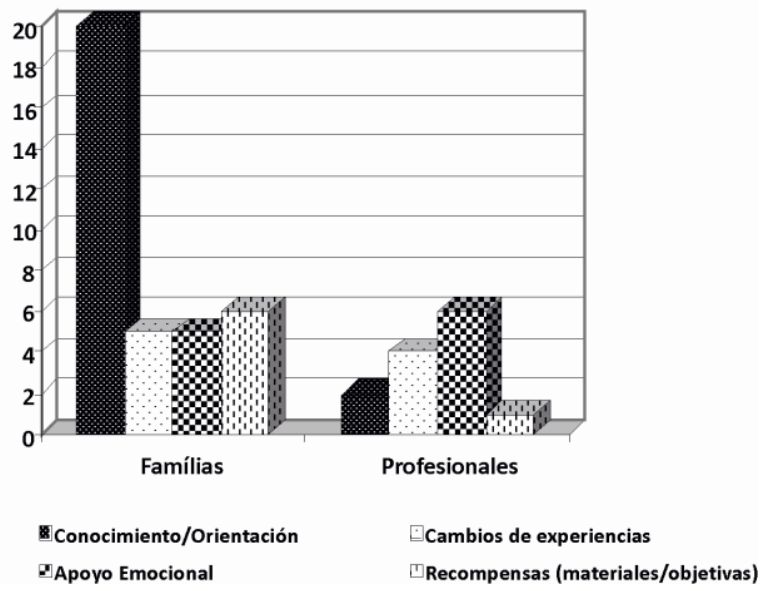

Figura 3. Aspectos positivos (puntos fuertes o fortalezas) de los servicios y de la atención familiar, en opinión de los familiares y de los profesionales.

La adquisición de conocimientos y las orientaciones e informaciones recibidas fueron los beneficios más citados por los participantes $(n=22)$ de ambos grupos (A y B), en especial aquellos referentes a cómo tratar al niño $(\mathrm{n}=7)$ - "Así, en términos de diálogo con los niños en ese rango, es excelente y, con ellos, es excelente la IC [institución]. Ellos hacen todo lo posible para ayudarte, para aconsejarte como tratar a los niños" (F7-pa) y para la estimulación e intervención con los niños en casa $(n=5)-$ " $A$ veces ayuda en casa, ¿verdad? Igual, dan algunas tareas prácticas para hacer en casa, que ayuda a desarrollar al niño, es eso" (F9-i).

El apoyo emocional (afectivo) fue considerado positivo porque favorece el hecho de compartir $(n=5)$ y promueve la autoestima $(n=6)$, tanto para el equipo técnico $(n=6)$, como para las madres $(\mathrm{n}=5)$ de ambos grupos (A y B), conforme ilustra la siguiente opinión: "Con seguridad, es bueno conversar con otras madres. Conversar con una madre, con otra, creo lo más importante de todo esto es que siempre piensas que tu problema es mayor que el del otro, y de repente la gente ve que no, que nuestro problema no es realmente mayor que el del otro" (F4-m).

Las recompensas materiales y objetivas $(n=7)$ fueron citadas, en especial por las madres $(n=6)$ de ambos grupos (A y B). Por ejemplo: la formación, la obtención del diploma, la amistad, o la actividad física. Anteriormente, el equipo técnico $(\mathrm{n}=1)$ mencionó las meriendas y las cestas básicas.

En relación a los profesionales (especialistas de los equipos técnicos) fueron evaluados como aspectos positivos: porque están disponibles e interesados $(n=6)$, atienden bien $(\mathrm{n}=5)$, evalúan las necesidades de los padres $(\mathrm{n}=3)$, atienden sin horario $(n=3)$, llaman por teléfono a los padres $(n=2)$, y conversan u orientan a los padres $(\mathrm{n}=2)$. La siguiente opinión ilustra el último aspecto positivo: "Estos profesionales están mucho más involucrados porque cada día nos están mostrando que la implicación de los padres hace mucho más que los resultados aquí. Los profesionales están mucho más interesados, cada vez. estimulan más a los padres a hacer el trabajo en casa" (P6-eq. téc.).

Observamos que los profesionales fueron considerados como accesibles e interesados en opinión de los propios directores de las instituciones $(\mathrm{n}=2)$ y de los especialistas del equipo técnico $(n=4)$. Por otro lado, los padres también evaluaron que los profesionales atienden bien $(n=3)$ y los responsables de las instituciones fueron los únicos que comentaron que los especialistas son los que realizan el apoyo de las necesidades de los familiares. En cuanto a que telefonearan a los padres fue visto como positivo por un niño - " $M e$ gusta. Mi padre trabaja y ella (la profesional) Ilama para que mi padre venga" (F1-c). Por tanto, la evaluación positiva en cuanto a los profesionales que trabajan en los servicios y en la atención familiar, fue mencionada, sobre todo por los profesionales. Por otra parte, no hubo diferencias en la percepción de las familias de los grupos A y B.

La participación de los progenitores en los servicios y en la atención familiar también fue evaluada positivamente por los profesionales $(n=4)$, además de la estructura física que fue otro aspecto evaluado como positivo $(n=3)$ por un padre, una madre y un responsable de la Institución.

En resumen, en cuanto a los aspectos positivos de los servicios y la atención familiar, se observa que para las familias el conocimiento/orientación y la información fue el beneficio más importante, mientras que para los profesionales fue el apoyo emocional. En general, las familias fueron las que evaluaron más positivamente los servicios y la atención familiar. Por otro lado, los profesionales fueron los que evaluaron de manera más positiva a los profesionales y la participación de los progenitores.

Aspectos negativos (debilidades) de los servicios y de las atenciones familiares. Los propios servicios y atenciones familiares fueron evaluados negativamente porque: (a) las familias, sobre todo las madres, permanecieron ociosas en la institución $(\mathrm{n}=11)$; (b) no son prioridad para la institución ( $\mathrm{n}=4)$; (c) no atienden las necesidades de las familias ( $\mathrm{n}=4)$; (d) ofrecen una escasa atención en grupo $(\mathrm{n}=3)$; (e) son muy informales $(\mathrm{n}=2)$; ( $\mathrm{f})$ no mejoran las condiciones de vida de la familia $(\mathrm{n}=2)$, ( $\mathrm{g})$ no favorecen la integración entre las madres $(n=1)$; y (h) no ofrecen transporte para los paseos $(n=1)$. La tabla 1 ilustra estos datos representado los respectivos participantes evaluados, si los familiares y/o los profesionales. 
Tabla 1. Aspectos negativos de los servicios y de la atención familiar, en opinión de los familiares y de los profesionales.

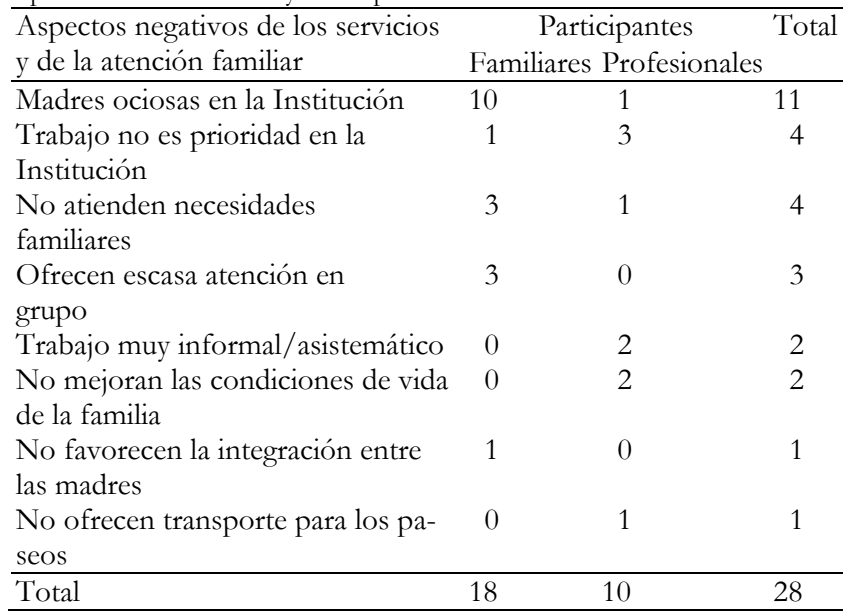

Se puede observar, que los familiares fueron quienes más evaluaron que se quedaban ociosos en la institución $(n=10)$, sobre todo las madres $(n=7)$, además de los padres $(n=2)$, de 1 hermano y de 1 profesional. Los familiares del grupo B fueron los que más citaron esta información $(n=6)$, lo que sugiere que los familiares de los niños menores de 6 años (grupo A) participan más de estos trabajos promovidos por las instituciones.

A pesar de ello, y de acuerdo con el equipo técnico $(n=3)$ y una madre, es un aspecto negativo el hecho de que las instituciones no dieran prioridad para implementar este tipo trabajo. Ejemplo: "Parece que tiene prioridad la atención al niño, la sensación que da es que si el niño no tiene atención...éste, está perdiendo el tiempo, esto es una tontería... Por tanto, no podemos ni siquiera abrir el calendario previsto en la agenda el día de las charlas, para que sea un día para las familias" (P1-eq.téc).

El hecho de que los Servicios y la Atención Familiar no atiendan las necesidades de las familias, sobre todo de las madres, fue comentado por 2 madres, 1 padre y 1 profesional. Es interesante destacar, que el trabajo fue considerado por los coordinadores técnicos, por un lado, como muy asistemático o informal. Al mismo tiempo, el trabajo fue evaluado como que no consiguió mejorar las condiciones de vida de las familias. Por lo tanto, aunque hay diferentes tipos de Servicios y de Atención Familiar que están siendo realizados por las instituciones, estos resultados muestran que éstos son insuficientes para atender a las madres, no consideran las necesidades de las familias y los tratamientos en grupo son escasos.

En cuanto a la evaluación negativa de la participación de las familias en los Servicios y la Atención Familiar, fueron consideradas escasas en relación con la institución $(n=12)$ y en la relación con el niño $(n=6)$. En relación con la institución porque: (a) no dan valor a lo que tienen $(n=4)$-en opinión de 2 madres y 1 especialista del equipo técnico y 1 gestor o responsable; (b) no comparecen en los servicios ofrecidos $(\mathrm{n}=5)$-en opinión de 1 madre, 2 coordinadores técnicos, 1 especialista del equipo técnico y 1 gestor o responsa- ble; (c) usan la discapacidad del niño para obtener ventajas $(n=2)$-en opinión de los especialistas del equipo técnico; y (d) hay más críticas en la percepción de las informaciones y orientaciones, en opinión de 1 especialista del equipo técnico. Luego, los profesionales, fueron quienes más negativamente evaluaron la participación de las familias en los Servicios y la Atención Familiar.

Estos datos indican que las familias son percibidas por los profesionales como pasivas y poco participativas; sin embargo, cuando demuestran un comportamiento contrario, o sea, cuestionando o reivindicando, son evaluadas de modo negativo. De esta forma, la visión que tienen las instituciones de los trabajos con las familias y de cómo ellas se organizan en esos trabajos parece reforzar la posición que los familiares, padres y madres tienen asumido, es decir, que es pasivo y dependiente. Los resultados siguientes corroboran esta situación.

En cuanto a la relación que mantiene la familia con los niños $(n=6)$, fue considerada por los profesionales y por las propias familias uno de los aspectos negativos de los Servicios y la Atención Familiar, apuntando que las familias: (a) no siguen las orientaciones y no cambian $(\mathrm{n}=4)$-en opinión de 2 madres, 1 coordinador técnico y 1 responsable; (b) hablan sobre el comportamiento negativo del niño -en opinión de 1 niño; y (c) crean ilusiones en cuanto al desarrollo del niño -en opinión de 1 profesional del equipo técnico.

Los profesionales también fueron considerados como aspectos negativos de los Servicios y la Atención Familiar. La calidad de las relaciones que mantienen con las familias fue considerada escasa $(n=8)$, sobre todo por los familiares $(\mathrm{n}=7)$, particularmente del grupo A $(\mathrm{n}=5)$. Por otro lado, la cantidad de profesionales en las instituciones fue considerada insuficiente $(n=8)$, por los propios profesionales, y en especial por los profesionales de los equipos técnicos $(n=7)$. Frente a esa información, se puede concluir que en las instituciones existe un número escaso de profesionales para atender la demanda de los familiares, y su habilidad interpersonal fue evaluada negativamente, en especial, por los familiares del grupo A (niños menores de 6 años).

La estructura física de las instituciones fue otro aspecto considerado negativo en la oferta de Servicios y de Atención Familiar. La cantidad de las salas fue vista como insuficiente para atender a los progenitores $(n=6)$, principalmente en la opinión de las madres y de los padres $(n=4)$, en especial del grupo $\mathrm{B}(\mathrm{n}=3)$, y de 2 especialistas de los equipos técnicos, que también evaluaron negativamente la estructura física.

Considerando las diferencias en las evaluaciones, según la perspectiva de las familias y de los profesionales, las familias fueron las que más negativamente evaluaron los Servicios y la Atención Familiar, destacando la ociosidad de las madres. Por otro lado, los profesionales fueron los que evaluaron más negativamente la participación de las familias en los Servicios y la Atención Familiar. A pesar de ello, la relación de la familia con el niño y el trabajo de los profesionales fueron evaluados como un aspecto negativo por las familias y por los profesionales. En la evaluación de los profesiona- 
les, las familias se referían más a la calidad (escasa) y los profesionales más a la cantidad (insuficiente).

Por tanto, los datos descritos en este estudio mostraron semejanzas y diferencias entre los familiares de los grupos A y $\mathrm{B}$, en lo que se refiere a la descripción y evaluación de los Servicios y la Atención Familiar. Las semejanzas se refieren a la participación en los grupos de padres, y a la evaluación positiva de los conocimientos/informaciones, apoyo emocional y recompensas materiales/objetivas obtenidas con la atención familiar. En relación con las diferencias, se percibe que el grupo A fue el que menos mencionó su participación en las atenciones informales/asistemáticas y de confraternización/fiestas. A pesar de ello, los familiares del grupo A fueron los que más negativamente evaluaron a los profesionales (calidad de la relación). Por otro lado, los familiares del grupo B fueron los que más mencionaron permanecer ociosos en la institución y los que más consideraron la estructura física de la institución como un aspecto negativo para las intervenciones familiares.

\section{Discusión y Conclusiones}

La literatura de este ámbito destaca, desde hace décadas, la importancia que tiene la participación de los familiares en los programas de atención a niños, sobre todo en los de intervención temprana (Aiello, 2004; Campos y Garcia, 2007). Los progenitores han sido reconocidos como elementos fundamentales para el proceso de aprendizaje y desarrollo de los niños desde 1930-1940, cuando los programas de intervención para las familias también comienzan a proliferar en los Estados Unidos (La Rossa, 1997, Pérez-López, 2004).

Por lo tanto, se han vislumbrado nuevas perspectivas en la atención familiar, aunque no necesariamente en todos los contextos y áreas de aplicación. A partir de 1950, tanto en los Estados unidos como en Brasil y España, surgieron los modelos preventivos de atención (prevención), resaltando una forma de comprender al hombre en su carácter histórico y social. Esa perspectiva teórica de trabajo con las familias ha sido considerada prometedora en el área de intervención temprana, puesto que crea oportunidades para que las personas ejerzan sus capacidades y desarrollen nuevas competencias, a la vez que concibe a los profesionales como agentes que ayudan a las familias y no actúan por ellas (Dunst et al., 2002).

Sin embargo, esta visión teórica no ha sido aplicada en la práctica de los profesionales y de las instituciones. En las instituciones investigadas por este estudio, esta no ha sido la visión predominante, pues los tipos de Servicios y de Atención Familiar que ofrecen las instituciones son característicos de una proposición de intervención denominada "para las familias" (Goldani, 2005), siendo lo contrario de un trabajo que es realizado con la participación activa y co-construida por la familia (Doherty, 2000).

En cuanto a la justificación por no ofrecer todos los Servicios y la Atención Familiar que podrían ser ofrecidos, los profesionales de las instituciones mencionan dificultades re- lacionadas con la institución o con las familias, señalando que los problemas o impedimentos se refieren a uno u otro responsable y no a una combinación de factores. Esta visión se caracteriza, sobre todo, por una relación de causa y efecto, idea que está en contraposición con el paradigma sistémico (Vasconcelos, 2002) y el paradigma de los apoyos (Aranha, 2001; MEC/SEESP, 2005), que fundamentan el proceso de inclusión social.

Las razones atribuidas a las dificultades de la institución para implementar trabajos con las familias parecen contradictorias con los valores que atribuyen a la participación de las mismas, pues los profesionales también comentan que son consideradas la base para la atención a los niños. En este caso, el incumplimiento de los Servicios y la Atención Familiar, especialmente en los familiares del grupo B, indica parte de esa falta de compromiso de las instituciones, y contradice la información de que la familia sea indispensable, o el principal factor de promoción del desarrollo del niño.

Estas cuestiones sugieren que el discurso predominante de que la familia es considerada importante para trabajo desarrollado con el niño, es superficial o está poco fundamentado, lo que implica en una agenda de trabajo que no es prioritario este tipo de servicio o atención. Sumado a las cuestiones ya consideradas como negativas en los Servicios y la Atención Familiar, la estructura física inadecuada también indica la falta de importancia que tiene la familia para las instituciones, aunque el discurso en ese aspecto sea el contrario.

A pesar de la evaluación negativa en relación con los Servicios y la Atención Familiar, fueron identificados algunos beneficios, especialmente, las orientaciones e informaciones en cómo tratar al niño, y en relación a la intervención con el niño en casa. Así mismo, se observa que aunque las instituciones de atención desconozcan las características de la estructura y del funcionamiento de las familias, esos servicios tienen atendida parte de sus necesidades, tal y como fue percibido por ellas. Al mismo tiempo, reforzaron la visión de que los padres son reproducciones de los profesionales en casa y solamente por esto ellos forman parte de la atención a los niños.

Muchos programas esperan que los padres asuman el papel integral de un co-terapeuta, implementando en casa las orientaciones recibidas por los profesionales de la salud y la educación (Bolsanello y Pérez-López, 2007; Fávero y Santos, 2005; Williams y Wishart, 2003). Con todo, este tipo de trabajo (co-terapia) fue característico de una fase inicial (de las instituciones de atención), cuya preocupación era, meramente, instrumental. Esto es, como una forma mediante la cual los padres podrían contribuir con los programas establecidos por los profesionales (Paniagua, 2004). Esta atención evolucionó hacia una visión más global e interactiva, en la cual se considera no sólo las necesidades del niño, sino de todo el grupo familiar (de Linares y Rodríguez, 2004). No obstante, no fue esto lo que los datos de la búsqueda constataron. Las intervenciones familiares se limitaban a las necesidades identificadas por los propios profesionales y se dirigían a las familias, o mejor aún, a las madres con el fin de entrenarlas en 
cuidados necesarios de los niños tales como la estimulación psicomotora, la comunicación (oral y escrita), y los límites y reglas para la convivencia cotidiana.

Es importante destacar que esa ampliación del foco de análisis es característica de un amplio movimiento que integra tanto el área específica de la atención temprana como la atención a personas con discapacidad, en general. Esta perspectiva de actuación considerada sistémica, bioecológica y transaccional de la atención temprana (Pérez-López, 2004, 2010) presenta valores y principios coherentes con el proceso de inclusión social, que demanda acciones centradas en la persona y en su familia/comunidad (MEC/SEESP, 2005). Por tanto, integrar a la familia, a la sociedad y al niño, como actores diferentes e interdependientes de ese proceso de intervención, más que de una tendencia contemporánea es una condición básica para los avances científicos del área (Bruder, 2010).

En cuanto a las intervenciones familiares, en este estudio, tienen como objetivo al niño y algunas veces, a la familia, tanto para el grupo A como para el grupo B, y ello explica el motivo de que las instituciones ofrezcan trabajos más centrados en el entrenamiento o educación de los progenitores. Los familiares del grupo A, por su parte, son aquellos, que más demandan una atención sistémica de las instituciones que proporcionan la atención, cuando sus hijos se encuentran en una fase temprana de su desarrollo. A pesar de todo, no fue posible observar ninguna distinción en los servicios y atenciones familiares ofrecidos, en función de la edad de los niños.

Lo ideal sería que los padres fuesen capacitados para una mejor relación con sus hijos y con los profesionales del área, formando así, un modelo de trabajo colaborativo entre progenitores y profesionales (Pérez-López, 2010), pues la relación con los profesionales fue uno de los aspectos peor eva-

\section{Referencias}

Aiello, A.L.R. (2004). Intervenção precoce centrada na família e formação de psicólogos: uma relação de parceria. Revista de Extensão e Pesquisa em Educação e Saúde, 2, 53-67.

Aranha, M.S.F. (2001). Paradigmas da relação da sociedade com as pessoas com deficiência. Revista do Ministério Público, 21, 160-173.

Bolsanello, M.A. y Pérez-López, J. (2007). Participación de las madres brasileñas en los servicios de atención temprana. Revista de Psicologia/International Journal of Developmental and Educational Psychology, 2, 383-393.

Brazelton, T.B. y Greenspan, S.I. (2002). As necessidades essenciais das crianças. Porto Alegre: Artes Médicas.

Bruder, M.B. (2000). Family-Centered early intervention: clarifying our values for the new millennium. Topics in early childhood special education, 20, 105-115.

Bruder, M.B. (2010). Early childhood intervention: a promise to children and families for their future. Exceptional Children, 76, 339-355.

Campos, C.E.A. y Garcia, J. (2007). Contribuições para a supervisão dos programas sociais com foco na família. Revista Katálysis, 10, 95-104.

de Linares, C. y Rodríguez, T. (2004). Bases de la intervención familiar en atención temprana. En J. Pérez-López y A.G. Brito de La Nuez (Dirs.), Manual de Atención Temprana (pp. 333-351). Madrid: Pirámide.

Dessen, M.A. (2009). Questionário de caracterização do sistema familiar. En L.N.D. Weber y M.A. Dessen (Dirs.), Pesquisando a família: instrumentos para coleta e análise de dados (pp. 102-114). Curitiba: Juruá luados por los familiares. Para eso, los profesionales deberían estar mejor preparados para incluir a las familias en la toma de decisiones sobre las metas que deberían ser establecidas en cuanto al desarrollo de los niños, y crear oportunidades para discutir y atender las necesidades de las madres o de otros familiares (Pérez-López, 2010). También, necesitan adaptarse a las demandas familiares, y actualizarse sobre las técnicas y procedimientos para una acción adecuada en esta área.

La atención a las familias de los niños con discapacidad debería estar guiada por una doble perspectiva. Esto es, proporcionar condiciones favorables para el desarrollo del niño y ayudar a cada familiar a enfrentarse a las dificultades de su condición de ser madre, padre, hermano o hermana de un niño con discapacidad (Omote, 2003). Esas lagunas, identificadas en los servicios y en la atención familiar, en el contexto brasileño, reflejan la falta de una política pública adecuada que ofrezca directrices orientadoras para esos diferentes contextos de desarrollo implementando acciones como esas.

Teniendo en cuenta la importancia de promover el desarrollo de los niños y de sus familias es necesario implementar cambios que sirvan para mejorar la calidad de la intervención familiar. Uno de estos cambios sería en el proceso de formación continua de los profesionales, especialistas de los equipos técnicos, coordinadores y responsables de las instituciones, para que todos puedan llevar a cabo intervenciones sistémicas, centradas en el niño, en su familia y en su comunidad, y que así consigan promover la capacitación de las familias brasileñas. En este sentido, el gobierno brasileño debería ofrecer políticas públicas, como el propio Programa de la Salud de la Familia -PSF, que optimicen ese proceso complejo de fortalecimiento de las familias brasileñas que tienen hijos con discapacidad o en situaciones de riesgo (Dessen y Cerqueira-Silva, 2008).

Dessen, M.A. y Cerqueira-Silva, S. (2008). Famílias e crianças com deficiência: em busca de estratégias para a promoção do desenvolvimento familiar. Anais do I Simpósio Nacional de Atenção e Estimulação Precoce, 39-58. Curitiba, Brasil.

Dessen, M.A. y Cerqueira-Silva, S. (2009). Desenvolvendo sistemas de categorias com dados de entrevistas. En L.N.D. Weber y M.A. Dessen (Dirs.), Pesquisando a família: instrumentos para coleta e análise de dados (pp. 43-56). Curitiba: Juruá.

Doherty, W.J. (2000). Family science and family citizenship: towards a model of community partnership with families. Family Relations, 49, 319-325.

Dunst, C. J. (2000). Revisiting "Rethinking early intervention." Topics in Early Childhood Special Education, 20, 95-104.

Dunst, C.J., Boyd, K., Trivette, C.M. y Hamby, D.W. (2002). Familyoriented program models and professional helpgiving practices. Family Relations, 51, 221-229.

Duvdevany, I. y Abboud, S. (2003). Stress, social support and well-being of Arab mothers of children with intellectual disability who are served by welfare services in northern Israel. Journal of Intellectual Disability Research, 47, 264-272.

Fávero , M.A.B. y Santos, M.A. (2005). Autismo infantil e estresse familiar: uma revisão sistemática da literatura. Psicologia: Reflexão e Crítica, 18, 358369.

Fundação Getúlio Vargas-FGV. (2010). Eventos. Classe média chega a 52\% da população. Obtido em: 
http://www.fgv.br/fgvportal/principal/idx_materia.asp?str_chave $=12$ $089 \& \operatorname{sessao}=2$

Goldani, A.M. (2005). Reinventar políticas para familias reinventadas: entre la "realidad" brasileña y la utopía. En Cambios de las familias en el marco de las transformaciones globales: necesidad de politicas públicas eficaces. Chile: CEPAL.

Gomes, V.F. y Bosa, C. (2004). Estresse e Relações Familiares na Perspectiva de Irmãos de Indivíduos com Transtornos Globais do Desenvolvimento. Estudos de Psicologia, 9, 553-561.

Kreutz, C.M. y Bosa, C.A. (2009). Intervenção precoce na comunicação pais-bebê com deficiência visual. Estudos de Psicologia, 26, 537-544.

La Rossa, R. (1997). The modernization of fatherhood. Chicago: University of Chicago Press.

Ministério da Educação/Secretaria de Educação Especial-MEC/SEESP. (2005). Saberes e práticas da inclusão - Avaliação para identificação das necessidades educacionais especiais. Brasília: Ministério da Educação.

Núñez, B. (2003). La Familia con un Hijo con Discapacidad: sus Conflictos Vinculares. Archives Argentinian of Pediatry, 101, 133-142.

Oliveira, M.R. (2007). Nascimento de filhos: rede social de apoio e envolvimento de pais e avós. Dissertação de Mestrado. Universidade de Brasília, Brasília/DF.

Oliveira, J.P. y Braga, T.M.S. (2004). Participação de familiares em práticas educativas de equipes multidisciplinares. Revista de Extensão e Pesquisa em Educação e Saúde, 2, 95-103.

Omote, S. (2003). A deficiência e a família. En M.C. Marquezine, M.A. Almeida, S. Omote y E.D.O. Tanaka (Dirs.), O papel da familia junto ao portador de necessidades especiais (pp. xv-xiii), Londrina: Eduel.

Paniagua, G. (2004). As famílias de crianças com necessidades educativas especiais. En C. Coll y A. Marchesi (Dirs.), Desenvolvimento psicológico e educação: transtornos de desenvolvimento e necessidades educativas especiais (pp.330346). Porto Alegre: Artmed.

Pereira-Silva, N.L. y Dessen, M.A. (2001). Deficiência mental e família: implicações para o desenvolvimento da criança. Psicologia: Teoria e Pesquisa, $17,133-141$.

Pereira-Silva, N.L. y Dessen, M.A. (2005). Intervenção precoce e família: contribuições do modelo bioecológico de Bronfenbrenner. En M.A. Dessen y A.L. Costa-Júnior (Dirs.), A ciência do desenvolvimento bumano: tendências atuais e perspectivas futuras (pp. 152-167). Porto Alegre: Artmed.

Pérez-López, J. (2004). Modelos explicativos del desarrollo aplicados a la atención temprana. En J. Pérez-López y A.G. Brito de La Nuez (Dirs.), Manual de Atención Temprana (pp. 27-44). Madrid: Pirámide.

Pérez-López, J. (2008). Da estimulação precoce à atenção precoce: novas alternativas de atuação profissional. Anais do I Simpósio Nacional de Atenção e Estimulação Precoce, 9-20. Curitiba, Brasil.

Pérez-López, J. (2010). Cambios conceptuales y marco teórico en atención temprana: nuevas necesidades de formación. Cadernos de Resumos do Sim pósio de Cooperação Binacional - Intervenção familiar e desenvolvimento infantil, 17-18. Bauru, Brasil.

Robbins, M., Szapocznik, J., Tejeda, M., Samuels, D., Ironson, G. y Antoni, M. (2003). The protective role of the family and social support network in a sample of Hiv- positive African American women: Results of a pilot study. Journal of Black Psychology, 29, 17-37.

Robles, Z. y Romero, E. (2011). Programas de entrenamiento para padres de niños con problemas de conducta: una revisión de su eficacia. Anales de Psicología, 27, 86-101.

Santos, E.F.R.R. (1993). Atendimento às famílias de deficientes: uma análise das propostas institucionais a partir dos relatos de profissionais que atuam na área. Dissertação de Mestrado, Universidade Federal de São Carlos, São Car$\operatorname{los} / \mathrm{SP}$.

Santos, P.L. y Graminha, S.S.V. (2005). Estudo comparativo das características do ambiente familiar de crianças com alto e baixo rendimento acadêmico. Paidéia, Cadernos de Psicologia e Educação, 15, 217-226.

Silva, A.F. y Elsen, I. (2006). Uma sociedade inclusivista para as famílias portadoras de membros com necessidade especial. Família, Saúde e Desenvol vimento, $8,154-162$.

Vasconcelos, M.J.E. (2002). Pensamento sistêmico: o novo paradigma da ciência. Campinas: Papirus.

Williams, K.R. y Wishart, J.G. (2003). The Son-Rise program intervention for autism: An investigation into family experiences. Journal of Intellectual Disability Research, 47, 291-299.

(Artículo recibido: 10-12-2010, revisión: 19-2-2012, aceptado: 22-2-2012) 